Atmos. Chem. Phys., 10, 3147-3153, 2010

www.atmos-chem-phys.net/10/3147/2010/

(C) Author(s) 2010. This work is distributed under

the Creative Commons Attribution 3.0 License.

\title{
Solar cycle signals in sea level pressure and sea surface temperature
}

\author{
I. Roy and J. D. Haigh \\ Department of Physics, Imperial College London, London, UK \\ Received: 29 October 2009 - Published in Atmos. Chem. Phys. Discuss.: 2 December 2009 \\ Revised: 25 March 2010 - Accepted: 26 March 2010 - Published: 31 March 2010
}

\begin{abstract}
We identify solar cycle signals in 155 years of global sea level pressure (SLP) and sea surface temperature (SST) data using a multiple linear regression approach. In SLP we find in the North Pacific a statistically significant weakening of the Aleutian Low and a northward shift of the Hawaiian High in response to higher solar activity, confirming the results of previous authors using different techniques. We also find a weak but broad reduction in pressure across the equatorial Pacific. In SST we identify a weak El Niñolike pattern in the tropics for the 155 year period, unlike the strong La Niña-like signal found recently by some other authors. We show that the latter have been influenced by the technique of compositing data from peak years of the sunspot cycle because these years have often coincided with the negative phase of the ENSO cycle. Furthermore, the date of peak annual sunspot number (SSN) generally falls a year or more in advance of the broader maximum of the 11-year solar cycle so that analyses which incorporate data from all years represent more coherently the difference between periods of high and low solar activity on these timescales. We also find that studies of the solar signal in SST over the second half of the 20th century may alias as ENSO signal if this is not properly taken into account.
\end{abstract}

\section{Introduction}

Signals of the 11-year solar cycle in various meteorological fields of the lower atmosphere, and in sea surface temperatures, have been presented by a number of authors. There is a consensus that any warming due to increased solar activity is not uniform, either within the atmosphere or at the ocean surface, but the patterns and amplitudes of the derived re-

Correspondence to: I. Roy

(i.roy06@imperial.ac.uk) sponses in temperature, and other fields, are still the subject of some uncertainty. In sea level pressure (SLP) the Aleutian Low tends to be weaker when the Sun is more active and that the Hawaiian High moves northwards (Christoforou and Hameed, 1997). A large response is found in the Pacific in boreal winter: a positive anomaly in the Bay of Alaska, consistent with Christoforou and Hameed results, and also a reduction in SLP near the date line around $20-40^{\circ} \mathrm{N}$ with a positive anomaly south of the equator (van Loon et al., 2007) (subsequently vL07). This has been interpreted as a strengthening of the SE trade winds crossing the equator, driving increased ocean upwelling and cooler equatorial temperatures (van Loon and Meehl, 2008).

This is consistent with the vL07 analysis of sea surface temperatures (SSTs) which shows a strong La Niña (Cold Event, CE)-like signal when the Sun is more active. However, another SST analysis (White et al., 1997) shows a slightly warmer band of water across the tropical Pacific associated with peaks in a decadal signal (DSO) identified as in phase with solar activity. A modeling and observational study suggests that coupling of changes in surface windstress to ocean circulation produces a Warm Event (WE) a few years after solar maximum (Meehl and Arblaster, 2009) (MA09). It also suggests that the White et al results might be interpreted as lagging solar maximum by $1-2$ years so that the results are not inconsistent. A more recent SST analysis (White and Liu, 2008), however, shows a phase-locking of harmonics of the ENSO time series with the solar cycle resulting in a WE-like signal for about 3 years around the peak of the DSO (with CEs approximately 2 years either side of the peak). Thus there is no clear picture of the SST response at solar maximum.

Any strong signal in SSTs would likely also be seen in near surface air temperature but two independent studies (Stott and Jones, 2009), using an optimal detection technique, and Lean and Rind (2008), using multiple regression analysis) both show very little solar signal in the tropics on centennial

Published by Copernicus Publications on behalf of the European Geosciences Union. 
timescales. They do, however, show bands of warming around mid-latitudes in both hemispheres, consistent with a response seen previously in 27 years of zonal mean tropospheric air temperatures (Haigh, 2003). In this paper we investigate further the solar signal in SLP and SSTs using a multiple regression technique. An important aspect of our paper is to point out that the timing is crucial to show how this produces apparent discrepancies between different analyses and how it may be used to test mechanisms proposed to explain solar-climate links, in the context of ENSO variability.

\section{Data and methodology}

We present results of a multiple linear regression analysis of over 150 years of SLP and SST data. The regressions are done for DJF data and all regression indices are used without time lags. For sea level pressures we use the Hadley Centre HadSLP2 dataset acquired from http://www.hadobs.org. This is an upgraded version of the monthly historical mean sea level pressure dataset HadSLP1, based on a compilation of numerous terrestrial and marine data over the time period 1850-2004 (Allan and Ansell, 2006).

We analyse two different sets of data for sea surface temperatures: one from the Hadley Centre and another from NOAA. The Hadley Centre dataset (HadSST2), obtained from http://hadobs.metoffice.com/hadsst $2 /$, is based on the recently created International Comprehensive Ocean Atmosphere Data Set (ICOADS) (Rayner et al., 2006). SST anomalies (relative to the mean values over 1961-1990) have been calculated over the globe between 1850 and 2004 . The other SST dataset we use is the NOAA extended reconstructed sea surface temperature data set (ERSST.v2) for 1854-2007 from http://www.cdc.noaa.gov/cdc/data.noaa. ersst.html. The two SST datasets differ in their data sources, in analysis procedure and the corrections algorithm applied before the 1940s. As sources, the NOAA product uses only in situ measurements while Hadley centre data include satellite-derived SST since early 1980s. The latter also include additional in situ observations from the UK Meteorological Office archive which are not included in the former (Vecchi and Soden, 2007). Vecchi et al. (2008) describe how inconsistencies of the various SST reconstructions in the equatorial eastern Pacific provide different results between the NOAA and HadISST data. We have carried out our analysis for both datasets but it did not alter our main results and here we present only results with the NOAA dataset.

The independent time-varying indices we use in the multiple regression are a linear trend (to simulate long-term climate change), stratospheric aerosol optical depth (mainly representing the influence of explosive volcanic eruptions), solar variability and ENSO. We employ a code which estimates amplitudes of variability due to these climate factors using an autoregressive noise model. In this methodology, noise coefficients (that might be present due to several unobserved sources) are calculated simultaneously with the components of variability so that the residual is consistent with a red noise model of order one. By this process it is possible to minimize noise being interpreted as signal. We then use a Student's t-test to measure the level of confidence of variability for different indices.

Stratospheric aerosol optical depth data are from http:// data.giss.nasa.gov/modelforce/strataer/tau_line.txt, which is up to 1999 . It has been extended to 2005 with near zero values. To represent the time variance of ENSO we use the Niño 3.4 index obtained from www.cpc.noaa.gov/data/indices.

For the solar influence we use monthly sunspot numbers (SSNs) available from ftp://ftp.ngdc.noaa.gov/STP/SOLAR_ DATA/SUNSPOT_NUMBERS/MONTHLY. Thus we include solar variability on solar cycle timescales but do not include the effect of any underlying long-term variations in solar irradiance. For statistical analysis of 155 years of data we are restricted for an indicator of solar activity to SSNs, TSI reconstructions or some geomagnetic indices. The focus of this paper is on 11-year cycle variability so that the accurate representation of longer term secular variability is less important. We have also carried out the regression using a number of TSI reconstructions (Solanki and Krivova, 2003; Hoyt and Schatten, 1993; Foster, 2004) and all produce similar solar cycle results but long term variations are not statistically separable from any climate change (linear trend or greenhouse gas) signal.

\section{Results}

The signal in SLP associated by the multiple regression analysis with solar cycle variability is presented in Fig. 1. It shows a region of positive anomaly, of up to $5 \mathrm{hPa}$, in the North Pacific with a smaller negative anomaly, magnitude around $0.5 \mathrm{hPa}$, in the equatorial Pacific. This pattern is robust to the inclusion or not of the ENSO index as an independent index in the regression analysis. It is consistent with the results of Christoforou and Hameed (1997) who showed that the Aleutian Low is weaker, and the Hawaian High positioned further north, during periods of higher solar activity. It agrees with vL07 in terms of the SLP signal around the north Pacific at peak SSN and continues throughout the following years of higher solar activity. It is also consistent with observational studies (Brönnimann et al., 2006; Haigh et al., 2005) and modeling studies (Haigh, 1996; 1999; Larkin et al., 2000; Matthes et al., 2006) which have indicated an expansion of the zonal mean Hadley cell, and poleward shift of the Ferrel cell, at solar maxima. It does not, however, reproduce the pattern found by vL07, using the HadSLP1 dataset, which placed a negative anomaly around $10-30^{\circ} \mathrm{N}$ in the mid-Pacific.

The solar cycle signal deduced in the NOAA SST data is presented in Fig. 2a. The pattern is essentially reproduced 


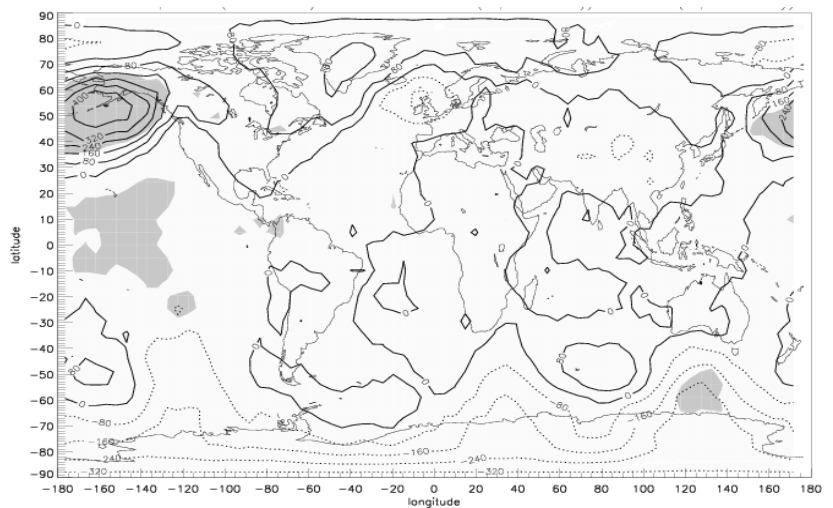

Fig. 1. The solar cycle signal in DJF sea level pressure (solar max solar min, Pa). The solar activity index used in the multiple regression analysis is sunspot number. Other independent parameters are a linear trend and indices of stratospheric aerosol optical depth and ENSO. Shaded regions are significant at the $95 \%$ level.

using the HadSST2 dataset and is robust to the inclusion or otherwise of an independent ENSO index. No regions of high statistical significance are detected but in the Eastern equatorial Pacific a tongue of warmer water is found, resembling a weak warm event. A stronger region of warming, of around $0.5 \mathrm{~K}$ occurs at $40^{\circ}$ latitude across the North Pacific, and more weakly in the South Pacific, with cooler regions equatorward in both hemispheres. The results in the North Pacific are qualitatively similar to those of vL07, although of smaller magnitude, but in the tropics they do not indicate the strong CE-like cooling found in the eastern Pacific in that paper. A multiple regression using only data over the second half of the 20th century, as used by White, et al. (1997), shows a strong El Niño WE-like pattern (Fig. 2b), but when we extend the data to 155 years this signal becomes much weaker. Furthermore, if we include an ENSO index in multiple regression of the latter period then the solar signal is also much reduced (not shown here), suggesting that the ENSO signal is being aliased onto the solar. This is consistent with the analysis of near surface air temperature by Lean and Rind (2008), Stott and Jones (2009) and Haigh (2003).

\section{Discussion}

To explain the apparent discrepancy between the different results, we investigate the different methodologies employed. vL07 deduced the solar signal by taking a composite of the data corresponding to the years identified with the peaks of the 14 solar activity cycles within the data period, and then associating the anomaly relative to the climatology with the effects of the Sun. The pattern was robust to the removal of data from either 1989 or 1905 , the only years identified as having a strong ENSO influence. (a)

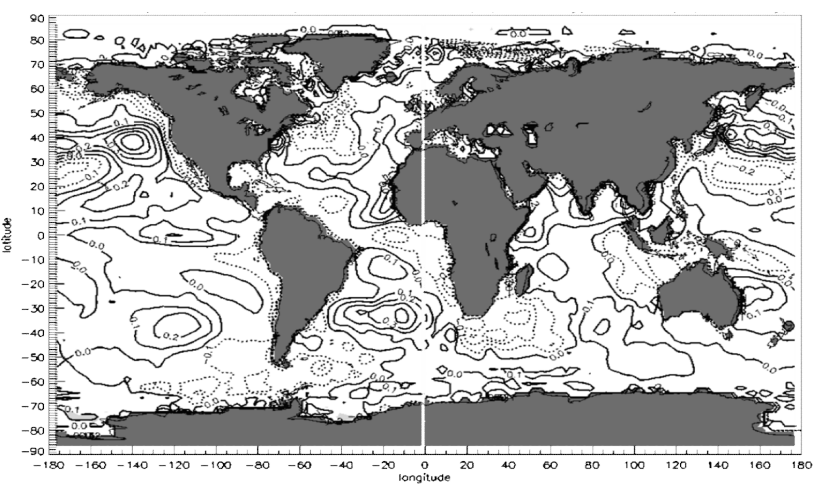

(b)

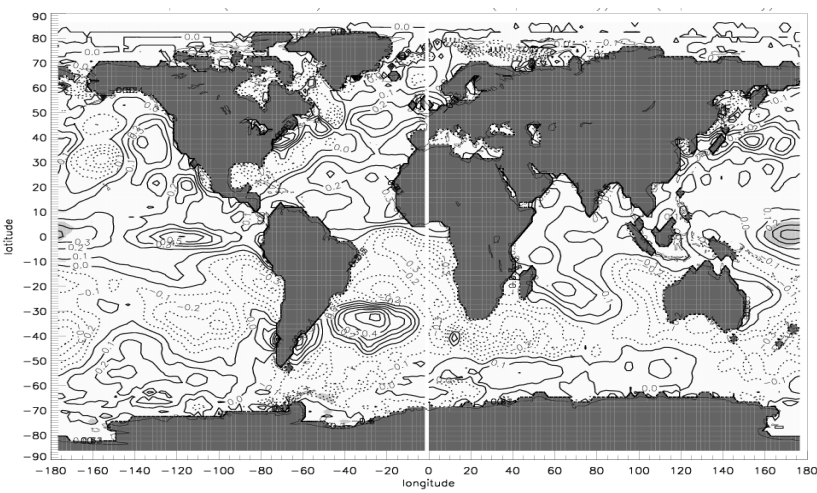

Fig. 2. (a) The solar cycle signal in DJF sea surface temperature (solar max - solar min, K) using the NOAA dataset for 155 years. The solar activity index used in the multiple regression analysis is sunspot number. Other independent parameters are a linear trend and indices of stratospheric aerosol optical depth and ENSO. Shaded regions (few and small) are significant at the $95 \%$ level. (b) As in (a) but using only data over the second half of the 20th century.

To investigate further any potential link between the apparent solar signal and ENSO, Fig. 3a presents a scatter plot of the values of these two parameters. No obvious relationship exists, and this is confirmed by a separate regression analysis of the ENSO time series (not shown). This would suggest that a signal identified with solar variability in tropical sea surface temperatures would be unlikely to express a particular phase of ENSO. When, however, solar cycle maximum years are identified, as shown in Fig. 3b, nine of the fourteen have a value of ENSO index lower than the average, and four of these years $(1893,1917,1989,2000)$ are associated with particularly strong cold events. Only one solar maximum year is associated with a significant positive ENSO signal and this, 1905, is a weak solar cycle. As it is only the solar maximum years that are used by vL07 to characterize the solar signal it is clear that their result will resemble a Cold Event (La Niña) pattern. It then remains to be determined to what extent the derived signal can be assigned as due to the Sun rather than mainly due to natural ENSO variability. 


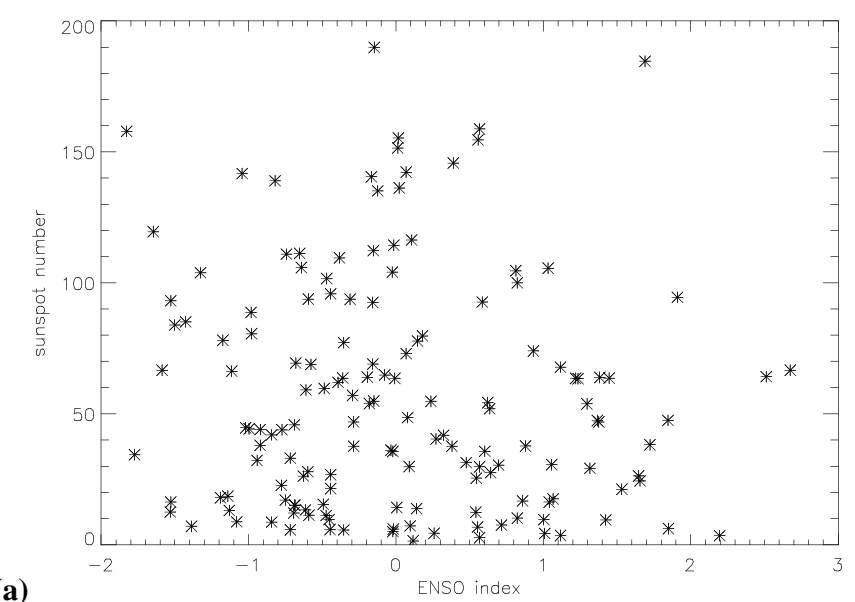

(a)

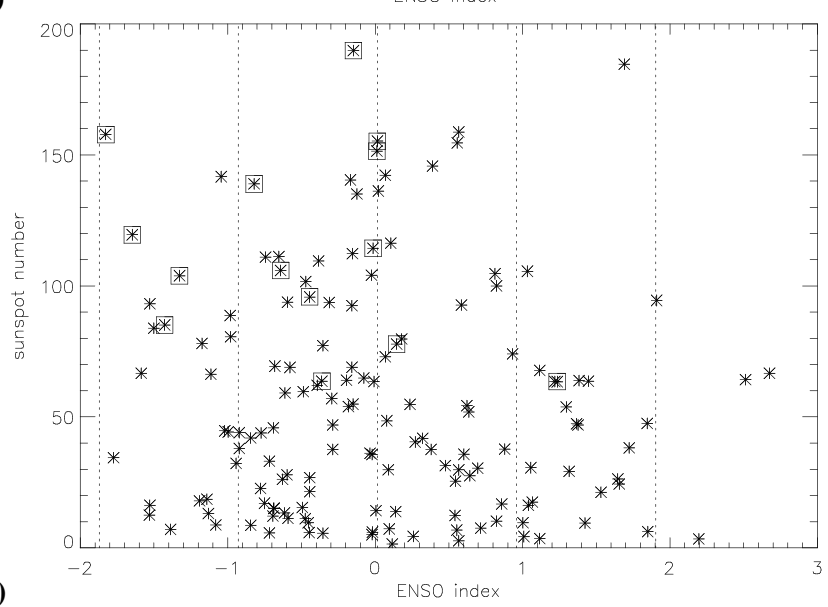

(b)

Fig. 3. (a) Scatter plot of ENSO index against sunspot number, DJF values from 1856 to 2007 inclusive. (b) As in (a) but years of solar cycle maximum are now identified with a box. Dashed vertical lines indicate the mean ENSO index and values at \pm 1 and 2 standard deviations from the mean.

Van Loon and co-authors have shown in multiple papers $(2007,2008,2009)$, that the response to peaks in solar forcing resembles La Niña conditions. van Loon and Meehl (2008) showed that the peak solar conditions are different from La Niña events in the Southern Oscillation mainly in the equatorial stratosphere. If this is the case, and the solar signal is not ENSO-like, then it would raise doubts with regard to any proposed mechanisms for a solar influence on climate which are based on ENSO-like atmosphereocean coupling and changes to the Walker cell etc. The differences are confined, however, to pressures less than $25 \mathrm{hPa}$, i.e., right at the top of the NCEP/NCAR Reanalysis Dataset domain.

It is not immediately obvious why Fig. 2 should show a weak WE-like response associated with higher SSNs rather than the CE pattern of vL07, and as suggested by Fig. 3. One possibility is that the multiple regression technique treats the solar and ENSO signals as linearly independent whereas, as
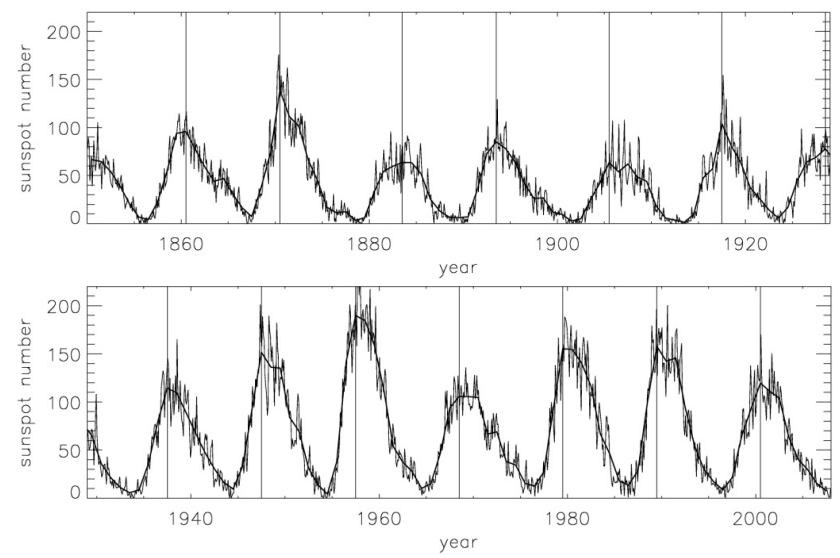

Fig. 4. Monthly mean sunspot number (thin curve) and the annual mean (thick curve). Vertical lines indicate the year of peak annual sunspot number for each solar cycle.

discussed by White and Liu (2008), there is non-linear coupling between the two influences, and that the latter cannot be cleanly separated from the former. However, this cannot be the whole story as the solar signal produced by the regression is essentially unaffected by the inclusion of an independent ENSO index and, furthermore, White and Liu (2008) show a WE pattern at the peak of the DSO with or without ENSO coupling. Rather, the answer appears to be related to the definition of peak solar activity.

Figure 4 shows the time series of monthly SSNs and of their annual means, it also identifies the years of highest annual SSN for each solar cycle, as used by vL07 to label solar peak years. It is apparent that peak years tend to occur very soon after the solar cycle becomes more active - this is true of all the stronger cycles - and at least a 1 year before a date that represents the peak of a broader decadal variation. Variations in TSI (e.g. Lean, 2000; Krivova et al, 2003) tend to follow the latter. Thus an analysis based on peak SSN years, such as the vL07 results, represents the solar signal at a particular phase of the solar cycle while Fig. 2, and the results of White et al. (1997), represent more broadly the difference between periods of higher and lower SSN. The work of White and Liu (2008) provides an indication as to why these may not give the same results: a WE signal near the peak of the DSO is typically preceded and followed, with a lead/lag of about 2 years, by a CE.

This difference in timing might also provide an explanation for other apparent discrepancies in solar signals. For example, in observational data van Loon et al. (2004) find a strengthening of the tropical Hadley cell when the Sun is more active while Kodera and Shibata (2006) find it weakened. Peak SSN composites are used in the former paper while correlations between meteorological data and the $10.7 \mathrm{~cm}$ solar activity index are used in the latter and thus, from the arguments presented above, they represent different 
aspects of solar cycle variability. Similarly in modeling studies: Meehl et al. (2008), with a transient model run analysed by peak SSN years, find a stronger Hadley circulation while Haigh (1996, 1999), using equilibrated solar maximum and minimum experiments, finds a weaker Hadley cell.

Our analysis provides some coherence to published results and may thus contribute to advance understanding of the associated physical process. One explanation for the solar response (MA09) requires that changes in absorption of solar radiation at the ocean surface initiate the earlier $\mathrm{CE}$ response. The processes involved would be akin to those proposed to be acting on centennial to millennial timescales, in which overall global warming increases meridional gradients in tropical sea surface temperatures, surface winds and shallow meridional overturning of the ocean (Clement et al., 1996). Paleoclimate studies (Emile-Geay et al., 2007) using idealised forcings in a simplified model have suggested that solar variability is manifest in similar overall changes in the tropical ocean circulations in response to longer term solar variability. Thus periods of higher insolation are statistically associated with an increased frequency of La Niña-like events (Mann et al., 2005). How the necessary changes in ocean circulation might become established within the timescale of the ascending portion of the solar cycle still needs to be established.

Meehl et al. $(2008,2009)$ discuss a peak in irradiance at the peak of the decadal solar oscillation producing the LaNina-like response. They show this is lagged after a year or two by an El-Nino-like event (although van Loon and Meehl (2008) show a weaker cold event at one year lag). We also find that there is often a cold event at peak SSN but this occurs a year or so in advance of the peak in irradiance so that the subsequent warm event coincides with the solar maximum in irradiance, as shown by White (2007) and White and Liu (2008). This difference in timing is crucial in considering mechanisms which involve forcing by solar irradiance.

Results from two different global coupled models (PCM and CCSM3) shown by Meehl et al. (2008), using multiple ensemble members from 20th century simulations and thus on the order of 40 to 50 realizations of peak solar forcing for each model, show a similar pattern to the shorter observational record, and Meehl et al. $(2008,2009)$ suggest mechanisms. Regarding the timing of forcing and responses, however, MA09 presented results showing that the result of these two models during DJF differ: PCM showed cooling starts one year before and reaches max at same year with warming around following year, whereas CCSM3 indicated warming one year before, cooling the same year and that cooling persists the following year. Such results are not consistent with the observational results of van Loon et al. (2008) who showed maximum cooling in peak SSN years, preceded and followed by lesser cooling (and no warming). The discrepancies between the two model results in terms of tropical Pacific SST mainly arise from the different ENSO variability within the models (MA09). In trying to disentangle the mechanisms for the apparent influences of the Sun on the climate of the lower atmosphere it has become useful to identify a "top-down" response from solar UV forcing in the stratosphere and a "bottom-up" response from solar warming of SSTs. Rind et al. (2008), using a model with various representations of stratospheric ozone, with and without a coupled slab ocean, study found that both proposed mechanisms are operative with the top-down effect contributing perhaps one half of the signal. They found a sea surface temperature response that produces the troposphere warming to be similar to that described by White et al. (1997) and very different from the La Niña response described by van Loon et al. (2007). In determining this SST signal Rind et al. (2008) compared years of higher solar irradiance with those of lower, using a threshold in UV irradiance, and would thus be more likely to find the broad DSO signal than the peak SSN signal, as discussed above.

Meehl et al. (2009) have also considered top-down and bottom-up effects in versions of three global climate models (CCSM3, WACCM-fixed-SST, WACCM-coupled), with mechanisms acting together or alone. They compared the results with the observations in both cases identifying the solar signal by the technique of solar maximum compositing. They found that with either forcing operating alone the signal in SSTs was weak but with both operating together the model produced a similar solar signal to that found in observations, viz. a La Niña-like response at peak SSN, followed after 1-2 years by a warm event-like signal. The solar signal in SLP appears more robust than that in SST but it raises another issue with regard to mechanisms. Associated with the (putative solar-induced) cold event in tropical SSTs, as is standard with an ENSO negative phase, is a positive anomaly in N. Pacific SLP in winter and Meehl et al. (2008) discuss how the two are physically related. In the observations, however, this positive anomaly in mid-latitudes is maintained for several years, and continues through the following "warm event" which would normally be accompanied by a negative SLP anomaly. This poses problems for any mechanism proposed for a solar influence on climate which involves an ENSO-type coupled ocean-atmosphere processes. Our analysis thus suggests that the response of SST to variations in solar irradiance and the mechanisms of this response remain to be established in the context of internal model (ENSO and other) variability and the uncertainties in timings discussed above.

\section{Summary}

We identify solar cycle signals in the North Pacific in 155 years of sea level pressure and sea surface temperature data. In SLP we find in the North Pacific a weakening of the Aleutian Low and a northward shift of the Hawaiian High in response to higher solar activity, confirming the results of previous authors using different techniques. We also find a 
broad reduction in pressure across the equatorial region but not the negative anomaly in the sub-tropics detected by vL07. In SST we identify the warmer and cooler regions in the North Pacific found by vL07 but instead of the strong Cold Event-like signal in tropical SSTs we detect a weak WE-like pattern in the 155 year dataset.

We find that the peak SSN years of the solar cycles have often coincided with the negative phase of ENSO so that analyses, such as that of vL07, based on composites of peak SSN years find a La Niña response. As the date of peak annual SSN generally falls a year or more in advance of the broader maximum of the 11-year solar cycle it follows that the peak of the DSO is likely to be associated with an El Niño-like pattern, as seen by White et al. (1997). An El Niño pattern is clearly portrayed in our regression analysis using only data from second half of the last century, but inclusion of ENSO as an independent regression index results in a significant diminution of the solar signal in tropical SST, showing further how an ENSO signal might be interpreted as due to the Sun.

Any mechanisms proposed to explain a solar influence should be consistent with the full length of the dataset, unless there are reasons to think otherwise, and analyses which incorporate data from all years, rather than selecting only those of peak SSN, represent more coherently the difference between periods of high and low solar activity on these timescales.

The SLP signal in mid-latitudes varies in phase with solar activity, and does not show the same modulation by ENSO phase as tropical SST, suggesting that the solar influence here is not driven by coupled-atmosphere-ocean effects but possibly by the impact of changes in the stratosphere resulting in expansion of the Hadley cell and poleward shift of the subtropical jets (Haigh et al., 2005). Given that climate model results in terms of tropical Pacific SST can be dependent on different ENSO variability within the models, our analysis indicates that the robustness of any proposed mechanism of the response to variations in solar irradiance needs to be analyzed in the context of ENSO variability where timing plays a crucial role.

Acknowledgements. Indrani Roy is supported by a UK Natural Environment Research Council postgraduate studentship. We are grateful to Myles Allen for provision of the multiple regression code. The NERC British Atmospheric Data Centre provided access to some of the datasets.

Edited by: D. Shindell

\section{References}

Allan, R. and Ansell, T.: A new globally complete monthly historical gridded mean sea level pressure dataset (HadSLP2): 18502004, J. Climate, 19(22), 5816-5842, 2006.

Brönnimann, S., Ewen, T., Griesser, T., and Jenne, R.: Multidecadal signal of solar variability in the upper troposphere during the 20th century, Space Sci. Rev., 125(1-4), 305-317, 2006.

Christoforou, P. and Hameed, S.: Solar cycle and the Pacific "centers of action”, Geophys. Res. Lett., 24(3), 293-296, 1997.

Clement, A. C., Seager, R., Cane, M. A., and Zebiak, S. E.: An ocean dynamical thermostat, J. Climate, 9(9), 2190-2196, 1996.

Emile-Geay, J., Cane, M., Seager, R., Kaplan, A., and Almasi, P., et al.: El Niño as a mediator of the solar influence on climate, Paleoceanography, 22, PA3210, doi:10.1029/2006PA001304, 2007.

Foster, S.: Reconstruction of solar irradiance variations, for use in studies of global climate change: application of recent SoHO observations with historic data from the Greenwich Observatory, $\mathrm{Ph} . \mathrm{D}$. Thesis, University of Southampton, Southampton, UK, 2004.

Haigh, J. D.: The impact of solar variability on climate, Science, 272(5264), 981-984, 1996.

Haigh, J. D.: A GCM study of climate change in response to the 11-year solar cycle, Q. J. Roy. Meteor. Soc., 125(555), 871-892, 1999.

Haigh, J. D.: The effects of solar variability on the Earth's climate, Philos. T. R. Soc. A., 361(1802), 95-111, 2003.

Haigh, J. D., Blackburn, M., and Day, R.: The response of tropospheric circulation to perturbations in lower-stratospheric temperature, J. Climate, 18(17), 3672-3685, 2005.

Hoyt, D. V. and Schatten, K. H.: A discussion of plausible solar irradiance variations, 1700-1992, J. Geophys. Res., 98(A11), 18895-18906, 1993.

Kodera, K. and Shibata, K.: Solar influence on the tropical stratosphere and troposphere in the northern summer, Geophys. Res. Lett, 33, L19704, doi:10.1029/2006GL026659, 2006.

Krivova, N. A., Solanki, S. K., Fligge, A., and Unruh, Y. C.: Reconstruction of solar irradiance variations in cycle 23: Is solar surface magnetism the cause?, Astronomy and Astrophysics, 399(1), L1-L4, doi:10.1051/0004-6361:20030029, 2003.

Larkin, A., Haigh, J. D., and Djavidnia, S.: The effect of solar UV irradiance variations on the Earth's atmosphere, Space Sci. Rev., 94(1-2), 199-214, 2000.

Lean, J.: Evolution of the sun's spectral irradiance since the Maunder Minimum, Geophys. Res. Lett., 27(16), 2425-2428, 2000.

Lean, J. L. and Rind, D. H.: How natural and anthropogenic influences alter global and regional surface temperatures: 1889 to 2006, Geophys. Res. Lett., 35, L18701, doi:10.1029/2008GL034864, 2008.

Mann, M. E., Cane, M. A., Zebiak, S. E., and Clement, A.: Volcanic and solar forcing of the tropical Pacific over the past 1000 years, J. Climate, 18(3), 447-456, 2005.

Matthes, K., Kurodo, Y., Kodera, K., and Langematz, U.: Transfer of the solar signal from the stratosphere to the troposphere: Northern winter, J. Geophys. Res.-Atmos., 111, D06108, doi:10.1029/2005JD006283, 2006.

Meehl, G. A., Arblaster, J. M., Branstator, G., and van Loon, H.: A coupled air-sea response mechanism to solar forcing in the Pacific region, J. Climate, 21(12), 2883-2897, 2008.

Meehl, G. A. and Arblaster, J. M.: A Lagged Warm Event-Like 
Response to Peaks in Solar Forcing in the Pacific Region, J. Climate., 22(13), 3647-3660, 2009.

Meehl, G. A., Arblaster, J. M., Matthes, K., Sassi, F., and van Loon, H.: Amplifying the Pacific Climate System Response to a Small 11-Year Solar Cycle Forcing, Science, 325, 1114-1118, doi:10.1126/science.117287, 2009.

Rayner, N. A., Brohan, P., Parker, D. E., and Folland, C. K., et al.: Improved analyses of changes and uncertainties in sea surface temperature measured in situ sice the mid-nineteenth century: The HadSST2 dataset, J. Climate, 19(3), 446-469, 2006.

Rind, D., Lean, J., Lerner, J., Lonergan, P., and Leboissitier, A.: Exploring the stratospheric/tropospheric response to solar forcing, J. Geophys. Res.-Atmos., 113, D24103, doi:10.1029/2008JD010114, 2008.

Solanki, S. K. and Krivova, N. A.: Can solar variability explain global warming since 1970?, J. Geophys. Res., 108(A5), 1200, doi:10.1029/2002JA009753, 2003.

Stott, P. A. and Jones, G. S.: Variability of high latitude amplification of anthropogenic warming, Geophys. Res. Lett., 36, L10701, doi:10.1029/2009GL037698, 2009. van Loon, H., Meehl, G. A. and Arblaster, J. M.: A decadal solar effect in the tropics in July-August, J. Atmos. Sol.-Terr. Phy., 66(18), 1767-1778, 2004.

van Loon, H., Meehl, G. A., and Shea, D. J.: Coupled airsea response to solar forcing in the Pacific region during northern winter, J. Geophys. Res.-Atmos., 112(D2), D02108, doi:10.1029/2006JD007378, 2007.

van Loon, H. and Meehl, G. A.: The response in the Pacific to the sun's decadal peaks and contrasts to cold events in the Southern Oscillation, J. Atmos. Sol.-Terr. Phy., 70(7), 1046-1055, 2008.

Vecchi, G. A. and Soden, B. J.: Global Warming and the Weakening of the Tropical Circulation, J. Climate., 20, 4316-4340, 2007.

Vecchi, G. A., Clement, A., and Soden, B. J.: Examining the tropical Pacific's response to global warming, Eos Trans. AGU, 89(9), 89(9) 81-83, doi:10.1029/2008EO090002, 2008.

White, W. B., Lean, J, Cayan, D. R., and Dettinger, M. D.: Response of global upper ocean temperature to changing solar irradiance, J. Geophys. Res.-Oceans, 102(C2), 3255-3266, 1997.

White, W. B. and Liu, Z. Y.: Non-linear alignment of El Nino to the 11-yr solar cycle, Geophys. Res. Lett., 35, L19607, doi:10.1029/2008GL034831, 2008. 